\title{
Fusion of HSP70 gene of Mycobacterium tuberculosis to hemagglutinin (H5) gene of avian influenza virus in DNA vaccine enhances its potency.
}

\begin{abstract}
A series of plasmids containing the HSP70 gene of Mycobacterium tuberculosis fused to the hemagglutinin (H5) gene of H5N1 avian influenza virus (AIV) (H5-HSP70 (heat shock protein 70) vaccine) or individual $\mathrm{H} 5$ gene (H5 vaccine) or HSP70 gene (HSP70 vaccine) were constructed based on the plasmid pcDNA3.1. Expression of H5 gene in Vero cells in vitro and in chickens in vivo was confirmed following their transfection and immunization with H5 or H5-HSP70 vaccines. Controls consisted of HSP70 vaccine, empty plasmid pcDNA3.1 and co-administered H5 and HSP70 vaccines. H5-HSP70 vaccine produced in chicken higher hemagglutination inhibition (HI) antibody titer than $\mathrm{H} 5$ vaccine. However, the increase was not statistically significant. We have demonstrated for the first time that the H5 DNA vaccine with fused HSP70 gene may produce an enhanced induction of humoral immune response to AIV in chickens.
\end{abstract}

Keyword: Avian influenza virus; Hemagglutinin H5; DNA vaccine; Humoral immunity. 